\title{
Thermonuclear Supernova Explosions from White Dwarfs in Different Progenitor Systems
}

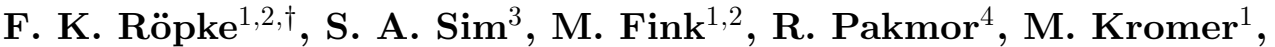 \\ I. R. Seitenzahl ${ }^{1,2}$, A. J. Ruiter ${ }^{2}$, and W. Hillebrandt ${ }^{2}$ \\ ${ }^{1}$ Universität Würzburg, Emil-Fischer-Str. 31, D-97074 Würzburg, Germany \\ ${ }^{2}$ Max-Planck-Institut für Astrophysik, Karl-Schwarzschild-Str. 1, D-85748 Garching, Germany \\ ${ }^{3}$ Research School of Astronomy and Astrophysics, Mount Stromlo Observatory, Cotter Road, \\ Weston Creek, ACT 2611, Australia \\ ${ }^{4}$ Heidelberger Institut für Theoretische Studien, Schloss-Wolfsbrunnenweg 35, D-69118 \\ Heidelberg, Germany \\ ${ }^{\dagger}$ email: fritz@mpa-garching.mpg.de
}

\begin{abstract}
Several progenitor scenarios have been suggested for Type Ia supernovae. Here we discuss the consequences for the explosion mechanism and for observables of some of them, which are explored by means of multi-dimensional hydrodynamic and radiation transfer simulations. While the observables predicted from delayed detonations of Chandrasekhar-mass white dwarfs agree reasonably well with the data, the corresponding progenitor systems may be too rare to account for the observed rate of Type Ia supernovae. Several alternatives are investigated of which violent mergers of two white dwarfs and, perhaps, double detonations of sub-Chandrasekhar mass white dwarfs hold promise for reproducing the observables of normal Type Ia supernovae.
\end{abstract}

Keywords. hydrodynamics - methods: numerical - stars: supernovae, white dwarfs

\section{Introduction}

Recent and ongoing transient searches discover a multitude of events that are attributed to thermonuclear explosions of (or on) white dwarfs. This extends the traditional picture of classical novae and Type Ia supernovae (SNe Ia). The class of SNe Ia, which was originally believed to be rather homogeneous, turned out to be surprisingly diverse. Apart from the $\sim 70 \%$ of normal events, there are about 3\% of SN2002cx-like, $9 \%$ of SN1991T-like and $18 \%$ of SN1991bg-like objects (Li et al. 2011). These emerging subclasses of SNe Ia are complemented with various peculiar sub- and super-luminous events. Does this diversity imply that there are several progenitor scenarios and/or explosion mechanisms realized in Nature?

One way of addressing this question is by forward modeling from an assumed progenitor over the explosion phase to the formation of observables. These predicted observables are then compared to observations of SNe Ia. In the following, different classes of explosion models are considered.

\section{Chandrasekhar-mass Explosions}

The most thoroughly explored scenario is the explosion of a WD at Chandrasekhar mass, $\sim 1.4 M_{\odot}$, because it seemed to provide a natural explanation for the assumed homogeneity of SNe Ia. In the light of the diversity of this class it is likely that such a 
scenario cannot account for all sub-classes and peculiar events. It may, however, explain the bulk of normal SNe Ia.

A prompt detonation of a Chandrasekhar-mass WD (Arnett 1969) burns the star completely at the high initial densities. Consequently, the ejecta consist almost exclusively of iron group elements (predominantly ${ }^{56} \mathrm{Ni}$ ), which is in conflict with observations. The intermediate-mass elements seen in the spectra of SNe Ia can only be synthesized if the burning proceeds partially at lower densities.

In contrast to supersonic detonations, deflagrations in WDs are mediated by thermal conduction of the degenerate electrons and are thus subsonic. Therefore, a combustion starting out in the deflagration mode brings the WD out of equilibrium and pre-expands the fuel material allowing for the synthesis of intermediate-mass elements. Laminar deflagration flames are too slow to catch up with the expansion of the star and the burning would be insufficient to power a SN Ia. Due to buoyancy instabilities, however, the flame does not stay laminar. Propagating from the WD's center outward, it produces an inverse density stratification in the gravitational field of the star. The Rayleigh-Taylor instability and secondary shear instabilities generate strong turbulence. Consequently, the flame interacts with turbulent eddies of various sizes and this accelerates the flame significantly.

The amount of burning and the energy release depend strongly on the way the flame is ignited. In Chandrasekhar-mass explosions a century of convective carbon burning precedes the actual flame ignition. Numerical simulations of this phase are extremely challenging due to its long duration and the high turbulence intensities involved (but see Höflich \& Stein 2002; Kuhlen et al. 2006; Zingale et al. 2009, for recent attempts). At the moment, the geometry of flame ignition is unclear and therefore different possibilities are considered. If ignited in many sparks around the center, the WD can be unbound (Röpke et al. 2006b, 2007). But even with a strong ignition, the asymptotic kinetic energy of the ejecta does not exceed $\sim 0.6 \times 10^{51} \mathrm{erg}$ and the ${ }^{56} \mathrm{Ni}$ production reaches at best about a third of a solar mass (Röpke et al. 2007). As the radioactive decay of ${ }^{56} \mathrm{Ni}$ powers the optical emission of SNe Ia, this translates into brightness of the modeled event. The most optimistic values for pure deflagrations in Chandrasekhar-mass WDs reach the fainter end of normal SNe Ia, but they cannot account for all of them. Moreover, the predicted spectra show peculiarities that can be attributed to a chemically mixed ejecta composition which is a natural consequence of the large-scale buoyancy instabilities in these models. For reproducing the spectra of normal SNe Ia, however, a stratified ejecta composition is required which turbulent deflagrations fail to produce. Branch et al. (2004) and Phillips et al. (2007) suggested the association of the SN 2002cx-like subclass of SNe Ia with turbulent deflagrations. Indeed, the shapes of spectra predicted from our simulations look qualitatively similar to those of SN 2002cx and SN 2005hk. The flux, however is generally too high if the ignition is assumed in a large number of sparks around the WD's center. An ignition in few sparks near the center may fail to unbind the WD. Spectra of these failed deflagrations, however, still look similar to those of SN 2002cx-like objects and the luminosity of the models becomes comparable to the observations.

The only chance for Chandrasekhar-mass explosion models to reach the ballpark of normal SNe Ia seems to be a detonation phase that follows burning in the deflagration mode. One way to realize this is the delayed detonation scenario (Khokhlov 1991), in which a spontaneous transition of the burning front from deflagration to detonation occurs in a late stage of the explosion.

The initial deflagration phase brings the Chandrasekhar-mass WD out of equilibrium and pre-expands the fuel material. Thus, part of the material processed by the detonation burns incompletely and produces intermediate-mass elements and oxygen. This leads to a clear chemical stratification in the outer layers of the ejecta. In addition, downdrafts of 
unburnt material left behind in the turbulent and unstable deflagration are now incinerated. The degree of the pre-expansion and thus the total ${ }^{56} \mathrm{Ni}$ production is determined by the energy release in the deflagration and by the delay between deflagration ignition and detonation triggering. The most important parameter is once again the geometry of flame ignition (but other parameters may also affect the strength of the deflagration phase, see e.g. Röpke et al. 2006a; Krueger et al. 2010; Jackson et al. 2010; Seitenzahl et al. 2011). The strongest pre-expansion and thus the weakest detonation is realized when igniting in many ignition sparks isotropically (Röpke \& Niemeyer 2007; Mazzali et al. 2007). This gives rise to a variability of ${ }^{56} \mathrm{Ni}$ production that leads to a range in brightnesses of the simulated events corresponding to that of normal SNe Ia. Delayed detonations, however, fail to account for subluminous objects.

The observables predicted from most of the simulations match the observations reasonably well as tested on a suite of two-dimensional models (Kasen et al. 2009), although no perfect agreement is reached (Blondin et al. 2011). However, the brightest and most asymmetric explosions in the Kasen et al. 2009 sample would not be classified as SNe Ia and do not correspond to any observed transients (Blondin et al. 2011, but see Maeda et al. 2010 for arguments favoring asymmetries). Interestingly, in this set of models, the correlation between peak luminosity in the $B$-band and the decline rate of the light curve (used to calibrate SNe Ia as distance indicators in observational cosmology, Phillips 1993; Phillips et al. 1999) was found to resemble that of the observations (Kasen et al. 2009).

Whether or not the delayed detonation scenario is a viable model for SNe Ia depends on the possibility of a deflagration-to-detonation transition to occur in WD combustion. Although some recent studies (e.g., Röpke 2007; Woosley 2007; Woosley et al. 2009; Poludnenko et al. 2011) indicate that this may indeed be the case, it is difficult to definitely decide on its realization in SNe Ia.

\section{Non-Chandrasekhar-mass Explosions}

From the explosion modeling point of view, delayed detonations of Chandrasekharmass WDs are quite successful. They cover the range of explosion energies and brightnesses of normal SNe Ia and reproduce their lightcurves and spectra relatively well. However, this scenario faces problems in explaining the occurrence rate of normal SNe Ia. The favored way of growing WDs to Chandrasekhar mass is the single-degenerate scenario, where the WD accretes from a main sequence or giant star until it reaches the limit. It is uncertain whether a thermonuclear explosion of a Chandrasekhar-mass object can emerge in a system of two merging WDs.

Some population synthesis calculations predict realization frequencies of the singledegenerate channel too low to account for the observed SN Ia rate (Ruiter et al. 2009). Moreover, the low X-ray flux from elliptical galaxies seems to be in conflict with a sufficient number of H-accreting progenitor systems (Gilfanov \& Bogdán 2010).

This indicates problems for the single-degenerate scenario, but, at present, it does not completely rule out Chandrasekhar-mass WD explosions as the main channel of normal SNe Ia. However, combined with the fact that the full range of peculiar events and subclasses of SNe Ia cannot be covered with delayed detonations of $M_{\mathrm{Ch}}$ WDs, it motivates the exploration of alternative models. Here, we consider two classes of such models: delayed detonations in differentially rotating WDs and violent mergers of two carbon-oxygen WDs. For a discussion of explosions in sub-Chandrasekhar-mass WDs we refer to the contribution of S. Sim et al. in this volume.

For some peculiar, super-bright SNe Ia (e.g., Howell et al. 2006; Taubenberger et al. 2011), total ejecta masses significantly in excess of $1.4 M_{\odot}$ and also very high ${ }^{56} \mathrm{Ni}$ masses 
have been claimed. A WD in equilibrium can support such masses only when rotating differentially. Explosions of such objects have been studied previously under the assumption of prompt detonations (Steinmetz et al. 1992; Pfannes et al. 2010a) and turbulent deflagrations (Pfannes et al. 2010b). Here we discuss a delayed detonation in a differentially rotating $W D$ of $2 M_{\odot}$. Angular momentum conservation and weaker gradients in the effective potential inhibit the growth of flame instabilities in lateral directions during the deflagration phase [similar to what has been found by Pfannes et al. (2010b) for pure deflagrations in such an object]. Thus, the initial deflagration flame propagates preferentially along the rotation axis. Consequently, burning in this phase is inefficient and the star does not significantly pre-expand. Therefore, when the detonation triggers, it finds copious amounts of unburned material at high densities which are burned into ${ }^{56} \mathrm{Ni}$. This gives rise to a bright and vigorous explosion. The kinetic energy of the ejecta is $1.96 \times 10^{51} \mathrm{erg}$. Although the high predicted luminosity qualifies the model for an explanation of super-luminous SNe Ia, it does not match important characteristics of these events (e.g., SN 2009dc, Taubenberger et al. 2011). Despite their extraordinary brightness, these objects typically show very low velocities in the spectral features of Si and S. In our model, such elements are seen at much higher velocities. Moreover, because the detonation reaches almost all of the material, only a tiny amount of carbon is left in the ejecta. This most likely is insufficient to produce the carbon features typically seen in the spectra of super-luminous objects.

Most population synthesis calculations agree that mergers of two CO WDs should be very common. The outcome of such mergers may be diverse and depends on the parameters of the merging system (mass of the primary WD, mass ratio). This parameter space has not been fully explored yet, and some parts of it are hard to access with numerical simulations. Some configurations may avoid thermonuclear explosion (e.g., Saio \& Nomoto 1998) and lead to the formation of a neutron star by gravitational collapse (Saio \& Nomoto 1985). For mass ratios close to unity, however, the mergers proceed dynamically and can be followed in simulations. Pakmor et al. (2010) studied the merger of two $0.9 M_{\odot}$ WDs. Here (as well as in some other cases, Pakmor et al. 2011) tidal interaction strongly deforms both stars and they merge violently. At the point where the two masses collide, thermodynamic conditions suitable for triggering a detonation are found. Such a detonation wave is able to incinerate the merged object almost completely. Although a total of $1.8 M_{\odot}$ is involved in the process, the produced ${ }^{56} \mathrm{Ni}$ mass is only of the order of $0.1 M_{\odot}$. The reason for this somewhat unexpected result is that in the violent merger the maximum density of the material does not increase. The highest density observed is that of the center of the $0.9 M_{\odot}$ WD. Burning at such densities does not reach nuclear statistical equilibrium. The resulting faint event matches characteristics of a SN Ia sub-class (the 1991bg-like objects) reasonably well (Pakmor et al. 2010). More massive WDs possess higher central densities and already a merger of a $1.1 M_{\odot} \mathrm{WD}$ with a $0.9 M_{\odot}$ WDs produces $0.64 M_{\odot}$ of ${ }^{56} \mathrm{Ni}$. Significant amounts of the total $2 M_{\odot}$ of material involved in this merger burn to intermediate-mass elements and to oxygen. In the ejecta, we find $0.47 M_{\odot}$ of oxygen and $0.09 M_{\odot}$ of carbon. Despite the large total mass of the exploding objects, the predicted lightcurves and spectra compare very favorably to that of normal SNe Ia.

\section{Conclusion}

At present it is impossible to decide on the viability of progenitor channels for SNe Ia from modeling the explosion of the emerging objects. For the bulk of normal SNe Ia, three possibilities come into question: Delayed detonations of Chandrasekhar-mass white 
dwarfs formed via the single-degenerate scenario (or perhaps also via double degenerates) agree well in their predicted observables with the astronomical data. Mergers of two rather massive carbon-oxygen WDs, however, also show very good agreement with the observations. This is perhaps not so much the case for double detonations of subChandrasekhar mass WDs in He-accreting systems, but such scenarios have also the potential of producing events that match the observations of normal SNe Ia to some degree (see the contribution of S. Sim et al. in this volume).

Breaking the degeneracy between these potential scenarios for normal SNe Ia is difficult. One possibility is to rank them according to their ability to explain the observed rate of these objects. Chandrasekhar mass explosions seem to be disfavored from this point of view (Ruiter et al. 2009), while sub-Chandrasekhar mass and double white dwarf merger models may come close to the expected rate (Ruiter et al. 2011). Although mergers in general are predicted to be common enough, the violent mergers discussed here are only a sub-class of them. They require rather massive WDs and restrict the parameter space further by imposing particular mass ratios between the merging objects. Whether these constraints still allow for a sufficiently high realization frequency to explain the rate of SNe Ia remains to be explored.

None of the models provides a perfect match with observed light curves or spectra, but this is certainly not expected given the fact that no fitting was attempted and that uncertainties in the modeling approaches remain. At the level of accuracy of our models it is difficult to favor or disfavor one particular of the three scenarios. There is, however, the possibility that observations in the infrared and UV bands or late-time spectra provide additional insights. Several complementary approaches have been explored to constrain progenitor channels leading to Chandrasekhar-mass explosions. An example that directly relates to the formation of the observables in the explosion ejecta is the search for material stripped from the companion when hit by the supernova blast wave (e.g., Marietta et al. 2000; Leonard 2007; Pakmor et al. 2008).

Deflagrations in Chandrasekhar-mass WDs may account for 2002cx-like objects, but perhaps only if they fail to completely unbind the star. Differential rotation increases the mass of the exploding object significantly beyond $1.4 M_{\odot}$ but it suppresses the deflagration efficiency. A delayed detonation in such differentially rotating WDs leads to very luminous and energetic explosions, but these do not match the observations of super-luminous SNe Ia nor any other objects known thus far. This may imply that the progenitors do not rotate differentially; however, rigid rotation is still a possibility.

\section{Acknowledgements}

This work was supported by the Deutsche Forschungsgemeinschaft via Emmy Noether Program (RO 3676/1-1), the Excellence Cluster EXC 153, and the Transregional Collaborative Research Center TRR 33. The work of FR is additionally supported by the ARCHES award 2011. We acknowledge that some of the results in this paper have been achieved using the PRACE Research Infrastructure resource JUGENE based in Germany at Forschungszentrum Jülich. In addition, computational resources for this project were provided by Forschungszentrum Jülich through the grants HMU13 and HMU14.

\section{References}

Arnett, W. D. 1969, ApESSS, 5, 180

Blondin, S., Kasen, D., Röpke, F. K., Kirshner, R. P., \& Mandel, K. S. 2011, MNRAS, 1228

Branch, D., Baron, E., Thomas, R. C., et al. 2004, PASP, 116, 903

Gilfanov, M. \& Bogdán, Á. 2010, Nature, 463, 924 
Höflich, P. \& Stein, J. 2002, ApJ, 568, 779

Howell, D. A., Sullivan, M., Nugent, P. E., et al. 2006, Nature, 443, 308

Jackson, A. P., Calder, A. C., Townsley, D. M., et al. 2010, ApJ, 720, 99

Kasen, D., Röpke, F. K., \& Woosley, S. E. 2009, Nature, 460, 869

Khokhlov, A. M. 1991, A\&\&A, 245, 114

Krueger, B. K., Jackson, A. P., Townsley, D. M., et al. 2010, ApJ, 719, L5

Kuhlen, M., Woosley, S. E., \& Glatzmaier, G. A. 2006, ApJ, 640, 407

Leonard, D. C. 2007, ApJ, 670, 1275

Li, W., Leaman, J., Chornock, R., et al. 2011, MNRAS, 412, 1441

Maeda, K., Benetti, S., Stritzinger, M., et al. 2010, Nature, 466, 82

Marietta, E., Burrows, A., \& Fryxell, B. 2000, ApJS, 128, 615

Mazzali, P. A., Röpke, F. K., Benetti, S., \& Hillebrandt, W. 2007, Science, 315, 825

Pakmor, R., Hachinger, S., Roepke, F. K., \& Hillebrandt, W. 2011, ArXiv e-prints

Pakmor, R., Kromer, M., Röpke, F. K., et al. 2010, Nature, 463, 61

Pakmor, R., Röpke, F. K., Weiss, A., \& Hillebrandt, W. 2008, A $\& A, 489,943$

Pfannes, J. M. M., Niemeyer, J. C., \& Schmidt, W. 2010a, A\& A, 509, A75+

Pfannes, J. M. M., Niemeyer, J. C., Schmidt, W., \& Klingenberg, C. 2010b, A\&A, 509, A74+ Phillips, M. M. 1993, ApJ, 413, L105

Phillips, M. M., Li, W., Frieman, J. A., et al. 2007, PASP, 119, 360

Phillips, M. M., Lira, P., Suntzeff, N. B., et al. 1999, AJ, 118, 1766

Poludnenko, A. Y., Gardiner, T. A., \& Oran, E. S. 2011, ArXiv e-prints

Röpke, F. K. 2007, ApJ, 668, 1103

Röpke, F. K., Gieseler, M., Reinecke, M., Travaglio, C., \& Hillebrandt, W. 2006a, A\&A, 453, 203

Röpke, F. K., Hillebrandt, W., Niemeyer, J. C., \& Woosley, S. E. 2006b, A\&AA, 448, 1

Röpke, F. K., Hillebrandt, W., Schmidt, W., et al. 2007, ApJ, 668, 1132

Röpke, F. K. \& Niemeyer, J. C. 2007, A\&广A, 464, 683

Ruiter, A. J., Belczynski, K., \& Fryer, C. 2009, ApJ, 699, 2026

Ruiter, A. J., Belczynski, K., Sim, S. A., et al. 2011, MNRAS, 1282

Saio, H. \& Nomoto, K. 1985, A\&A, 150, L21

Saio, H. \& Nomoto, K. 1998, ApJ, 500, 388

Seitenzahl, I. R., Ciaraldi-Schoolmann, F., \& Röpke, F. K. 2011, MNRAS, in preparation

Steinmetz, M., Muller, E., \& Hillebrandt, W. 1992, A\&A, 254, 177

Taubenberger, S., Benetti, S., Childress, M., et al. 2011, MNRAS, 412, 2735

Woosley, S. E. 2007, ApJ, 668, 1109

Woosley, S. E., Kerstein, A. R., Sankaran, V., Aspden, A. J., \& Röpke, F. K. 2009, ApJ, 704, 255

Zingale, M., Almgren, A. S., Bell, J. B., Nonaka, A., \& Woosley, S. E. 2009, ApJ, 704, 196 